\title{
Acute Bacterial Prostatitis
}

National Cancer Institute

\section{Source}

National Cancer Institute. Acute Bacterial Prostatitis. NCI Thesaurus. Code C92957.

An acute infection of the prostate gland caused by bacteria, most often Escherichia coli, Proteus mirabilis, Klebsiella, and Pseudomonas aeruginosa. Signs and symptoms include fever, lower back pain, urinary frequency, and painful urination. The urinalysis reveals the presence of white cells. Risk factors include intraprostatic ductal reflux, phimosis, urinary tract infections, and unprotected anal intercourse. 